ELECTRONIC RESEARCH ANNOUNCEMENTS OF THE AMERICAN MATHEMATICAL SOCIETY

Volume 7, Pages 79-86 (September 28, 2001)

S $1079-6762(01) 00097-\mathrm{X}$

\title{
ON PAIRS OF METRICS INVARIANT UNDER A COCOMPACT ACTION OF A GROUP
}

\author{
S. A. KRAT
}

(Communicated by Richard Schoen)

\begin{abstract}
Consider two intrinsic metrics invariant under the same cocompact action of an abelian group. Assume that the ratio of the distances tends to one as the distances grow to infinity. Then it is known (due to D. Burago) that the difference between the metric functions is uniformly bounded.

We will prove an analog of this result for hyperbolic groups, as well as a partial generalization of this result for the Heisenberg group: a word metric on the Heisenberg group lies within bounded GH distance from its asymptotic cone.
\end{abstract}

\section{INTRODUCTION}

Let $M$ be a set provided with two interior metrics $d_{1}$ and $d_{2}$. Assume that a group $G$ acts cocompactly on $M$ by isometries with respect to both metrics and

$$
\lim _{d_{2}(x, y) \rightarrow \infty} \frac{d_{1}(x, y)}{d_{2}(x, y)}=1 .
$$

Due to a result of D. Burago 2, if the group $G=\mathbb{Z}^{n}$, then there is a constant $C$ such that $\left|d_{1}(x, y)-d_{2}(x, y)\right| \leq C$. This fact means that all metrics on $M$ diverge linearly or stay within a finite distance from each other. Burago raised the question for which groups the same statement could be true. He suggested two different directions. The first is the case of semi-hyperbolic groups, i.e., groups of isometries of a space whose curvature is bounded from above by 0 . The other one is the case of nilpotent groups and first of all, the Heisenberg group. Our goal is to answer some of these questions.

The first part of the paper is devoted to the case of hyperbolic groups. To the best of our knowledge this result has never been published, even though many experts probably believe in it.

In some cases the fact that two metrics cannot diverge more slowly than linearly could be described as the finiteness of the Gromov-Hausdorff distance between the group with induced metric and its asymptotic cone. In the case of the abelian group $\mathbb{Z}^{n}$ the asymptotic cone is $\mathbb{R}^{n}$ and it lies within a finite Gromov-Hausdorff distance from $\mathbb{Z}^{n}$. We will describe one more case when this is true, and for the same reason, in the second part of this paper. In the case of hyperbolic groups the result does

Received by the editors February 16, 2001.

2000 Mathematics Subject Classification. Primary 51K05; Secondary 53C99.

Key words and phrases. Metric space, group action. 
not depend on the distance to the asymptotic cone because the asymptotic cone is not the Gromov-Hausdorff limit of the group with corresponding metrics.

The second part reviews the case of the simplest nontrivial infinite discrete nilpotent group, namely the discrete Heisenberg group $\Gamma$, where we get a partial result (for word metrics). The proof of the finiteness of the Gromov-Hausdorff distance between $\Gamma$ and its asymptotic cone was published in [7]. Here we will give the main idea of the proof and will show how the result follows from this fact.

The next natural case which generalizes the cases of abelian and hyperbolic groups is the case of semi-hyperbolic groups. It is not known yet whether the statement is true or false for it.

\section{Hyperbolic GROUP}

Let a hyperbolic group $G$ act cocompactly on a $\delta$-hyperbolic metric space $M$ by isometries with respect to two interior metrics $d_{1}$ and $d_{2}$.

Theorem 1.1. Assume that

$$
\lim _{\max \left(d_{1}(x, y), d_{2}(x, y)\right) \rightarrow \infty} \frac{d_{1}(x, y)}{d_{2}(x, y)}=1 ;
$$

then there is a constant $C$ such that $\left|d_{1}(x, y)-d_{2}(x, y)\right| \leq C$ for each $x, y \in M$.

Notation 1.2. We will denote the diameter of the compact set $M / G$ by $D$ and the difference $d_{1}(x, y)-d_{2}(x, y)=\Delta(x, y)$. In this paper by a geodesic we will mean the shortest path and consider only geodesics with natural parameterization.

The next lemma shows that the geodesic in metric $d_{2}$ lies in the constant neighborhood from the geodesic in metric $d_{1}$ with the same endpoints, where the constant does not depend on the choice of geodesics or their endpoints. This fact follows from the Morse lemma since under the conditions of the theorem any geodesic in metric $d_{2}$ is a quasi-isometric map in the metric $d_{1}$.

Lemma 1.3. Under the conditions of the theorem there exists a constant $c_{1}$ such that for each geodesic $\gamma_{2}:\left[0, l_{2}\right] \rightarrow\left(M, d_{2}\right)$ with endpoints $x$ and $y$ there is a geodesic $\gamma_{1}:\left[0, l_{1}\right] \rightarrow\left(M, d_{1}\right)$, with endpoints $x$ and $y$, such that $\gamma_{2}$ lies in the $c_{1}$-neighborhood (in the metric $d_{1}$ ) of $\gamma_{1}$.

Corollary 1.4. Since the conditions of Lemma 1.3 are symmetric in $d_{1}$ and $d_{2}$, there exists a constant $c_{2}$ such that for each geodesic $\gamma_{1}:\left[0, l_{1}\right] \rightarrow\left(M, d_{1}\right)$ with endpoints $x$ and $y$ there is a geodesic $\gamma_{2}:\left[0, l_{2}\right] \rightarrow\left(M, d_{2}\right)$, with endpoints $x$ and $y$, such that $\gamma_{1}$ lies in the $c_{2}$-neighborhood (in the metric $d_{2}$ ) of $\gamma_{2}$.

From Corollary 1.4 it follows that in the $c_{2}$-neighborhood (in the metric $d_{2}$ ) of any point on the geodesic $\gamma_{1}$ there is a point of the geodesic $\gamma_{2}$. Using triangle inequalities for these two points and the endpoints of the geodesics $\gamma_{1}$ and $\gamma_{2}$ one can derive the quasi-additivity property of the function $\Delta$.

Lemma 1.5. If $M$ is as described in the theorem and $\gamma_{1}:\left[0, l_{1}\right] \rightarrow M$ is geodesic in the metric $d_{1}$, then for each $t_{1} \in\left[0, l_{1}\right]$,

$$
\left|\Delta\left(\gamma_{1}(0), \gamma_{1}\left(l_{1}\right)\right)-\Delta\left(\gamma_{1}(0), \gamma_{1}\left(t_{1}\right)\right)-\Delta\left(\gamma_{1}\left(t_{1}\right), \gamma_{1}\left(l_{1}\right)\right)\right| \leq 2 c_{2}
$$

Now let us reformulate the conditions of the theorem in a more convenient form. 
Lemma 1.6. Under the conditions of the theorem

$$
\lim _{d_{1}(x, y) \rightarrow \infty} \frac{\Delta(x, y)}{d_{1}(x, y)}=0 .
$$

Since the group $G$ acts cocompactly on $M$, it is enough to consider the points of one orbit under the action of $G$ and measure the distances between them. We introduce a notation.

Notation 1.7. We choose a point $x_{0} \in M$, and let $G\left(x_{0}\right)$ be the orbit of this point under the action of the group $G$. For each $g \in G$, we will denote by $\gamma_{g}$ the geodesic in the metric $d_{1}$ with endpoints $x_{0}$ and $g\left(x_{0}\right)$, and by $\Delta(g)$, the quantity $\Delta\left(x_{0}, g\left(x_{0}\right)\right)$. It is easy to see that $\Delta(g)=\Delta\left(g^{-1}\right)$ for each $g$ in $G$.

Reasoning by contradiction we need to show that if the function $\Delta(x, y)$ is unbounded, then

$$
\lim _{d_{1}(x, y) \rightarrow \infty} \frac{\Delta(x, y)}{d_{1}(x, y)} \neq 0 .
$$

The $\delta$-hyperbolicity of the space $M$ means that if two geodesics with the same endpoint diverge relatively fast, then the distance between the other endpoints of these geodesics is almost the sum of the lengths of these geodesics. Now we need to define what we mean by that two geodesics $\gamma_{g_{1}}$ and $\gamma_{g_{2}}$ diverge relatively fast. The following function will show the moment after which the distance from each point on the geodesic $\gamma_{g_{1}}$ to geodesic $\gamma_{g_{2}}$ is greater than a certain $\epsilon$.

Definition 1.8. Let us define the function

$$
f\left(g_{1}, g_{2}, \epsilon\right)=\max \left\{t \mid d_{1}\left(\gamma_{g_{1}}(t), \gamma_{g_{2}}\right) \leq \epsilon\right\},
$$

where $g_{1}, g_{2} \in G$ and $\epsilon \in \mathbb{R}$. Here by $d_{1}\left(\gamma_{g_{1}}(t), \gamma_{g_{2}}\right)$ we mean the distance from the point $\gamma_{g_{1}}(t)$ to the set $\gamma_{g_{2}}$.

The function $f$ is not symmetric under the interchange of $g_{1}$ and $g_{2}$. But from the triangle inequalities it follows that the difference of values $f\left(g_{1}, g_{2}, \epsilon\right)$ and $f\left(g_{2}, g_{1}, \epsilon\right)$ is small.

Lemma 1.9. If $f$ is a function described above, then $\left|f\left(g_{1}, g_{2}, \epsilon\right)-f\left(g_{2}, g_{1}, \epsilon\right)\right| \leq \epsilon$.

The function $f\left(g_{1}, g_{2}, \epsilon\right)$ shows us the last time when the geodesic $\gamma_{g_{1}}$ came to the distance $\epsilon$ from the geodesic $\gamma_{g_{2}}$. But there remains the question whether the geodesic $\gamma_{g_{1}}$ stayed near the geodesic $\gamma_{g_{2}}$ before that? The answer is yes: it stays within the distance $\epsilon+\delta$. This fact follows from the $\delta$-hyperbolicity of $M$.

Lemma 1.10. Let $g_{1}, g_{2} \in G$. If $t \leq f\left(g_{1}, g_{2}, \epsilon\right)$, then $d_{1}\left(\gamma_{g_{1}}(t), \gamma_{g_{2}}\right) \leq \epsilon+\delta$.

The next lemma is some sort of analog of behavior of the angles in $\mathbb{R}^{3}$. If the angle between two rays with the same starting point in $\mathbb{R}^{3}$ is $2 \epsilon$, then each ray with the same starting point forms the angle at least $\epsilon$ with one of them. The angle between two rays in $\mathbb{R}^{3}$ shows how fast these rays diverge.

In our case we can say that if two geodesics diverge fast to the distance $2 \epsilon+2 \delta$, then each geodesic with the same starting point quickly moves $\epsilon$ units away from one of them. We cannot get $2 \epsilon$ instead of $2 \epsilon+2 \delta$, as in the case of rays, because the geodesics in our case can diverge and then converge again. But the amount of this divergence is bounded by $\epsilon+\delta$ in view of Lemma 1.10. 
Lemma 1.11. If $g_{1}, g_{2} \in G, C \in \mathbb{R}$ and $f\left(g_{1}, g_{2}, 2 \epsilon+2 \delta\right) \leq C$, then for each $g_{3} \in G$ either $f\left(g_{3}, g_{1}, \delta\right) \leq C+\epsilon$ or $f\left(g_{3}, g_{2}, \delta\right) \leq C+2 \delta+\epsilon$.

Now we assume that it is possible to find two elements $h_{1}$ and $h_{2}$ of the group $G$ such that the two geodesics connecting $x_{0}$ with $h_{1}\left(x_{0}\right)$ and $h_{2}\left(x_{0}\right)$ diverge relatively fast and the values $\Delta\left(x_{0}, h_{1}\left(x_{0}\right)\right)$ and $\Delta\left(x_{0}, h_{2}\left(x_{0}\right)\right)$ are relatively large. The proof of the existence of these elements is based on the almost additivity of the function $\Delta$ and we will give it later.

Using the elements $h_{1}, h_{2}, h_{1}^{-1}, h_{2}^{-1}$ we will construct the sequence of elements $\left\{g_{i}\right\}$ such that the geodesics connecting $x_{0}$ with $g_{i}\left(x_{0}\right)$ and $g_{i+1}\left(x_{0}\right)$ also diverge relatively fast and the $\Delta\left(g_{i}\left(x_{0}\right)\right)$ are relatively big. Then we will construct the piece wise geodesic path which starts at a point $x$ and then goes consecutively through all the points $x_{i}=g_{i} \circ \cdots \circ g_{1}\left(x_{0}\right)$. It consists of the isometric images of geodesics connecting $x_{0}$ with $h_{1}\left(x_{0}\right)$ and $h_{2}\left(x_{0}\right)$. From Lemma1.11 it follows that the geodesics connecting $x_{i}$ with $x_{0}$ and $x_{i+1}$ diverge relatively fast.

Lemma 1.12. If there exist $h_{1}, h_{2} \in G$ such that

$$
\min \left(\Delta\left(h_{1}\right), \Delta\left(h_{2}\right)\right) \geq 4 f\left(h_{1}, h_{2}, 6 \delta\right)+20 \delta
$$

then there exists a sequence $g_{1}, g_{2}, g_{3}, \ldots$ of elements of the group $G$ such that

$$
\Delta\left(g_{i}\right) \geq 4 f\left(\left(g_{1} \circ \cdots \circ g_{i-1}\right)^{-1}, g_{i}, 2 \delta\right)+4 \delta .
$$

Now that we have constructed the sequence $\left\{g_{i}\right\}$, we are going to prove that its existence contradicts the conditions of the theorem. It follows from the $\delta$ hyperbolicity of the space that the geodesic connecting $x_{0}$ and $x_{i}$ goes near all the points $x_{n}$ where $n<i$. This fact provides that $\Delta\left(x_{0}, x_{i}\right)$ and $d_{1}\left(x_{0}, x_{i}\right)$ are proportional to $i$. Then we have that

$$
\lim _{i \rightarrow \infty} \frac{\Delta\left(x_{0}, x_{i}\right)}{d_{1}\left(x_{0}, x_{i}\right)} \neq 0
$$

This implies that

$$
\lim _{i \rightarrow \infty} \frac{d_{1}\left(x_{0}, x_{i}\right)}{d_{2}\left(x_{0}, x_{i}\right)} \neq 1
$$

Lemma 1.13. Under the conditions of Lemma1.12,

$$
\lim _{\max \left(d_{1}(x, y), d_{2}(x, y)\right) \rightarrow \infty} \frac{d_{1}(x, y)}{d_{2}(x, y)} \neq 1 .
$$

Thus, we get a contradiction with the conditions of the theorem. Now the only thing that remains to be proved is the existence of two elements $h_{1}$ and $h_{2}$ such that the corresponding geodesics diverge fast and $\Delta\left(h_{1}\right)$ and $\Delta\left(h_{2}\right)$ are large.

Under the conditions of the theorem, the distances $d_{1}$ and $d_{2}$ cannot be small when the function $\Delta$ is large. It is obvious that at least one of them must be large. The other will also be large, since their quotient tends to 1 when the distances are large.

Lemma 1.14. Under the conditions of the theorem, there exists a constant $D_{0}$ such that if $\Delta(x, y)>D_{0}$ then $d_{1}(x, y)>\frac{1}{2} \Delta(x, y)$ and $d_{2}(x, y)>\frac{1}{2} \Delta(x, y)$.

Notation 1.15. If the conditions of the theorem hold, then the set

$$
\left\{d_{2}(x, y) \mid d_{1}(x, y) \leq \epsilon\right\}
$$

is bounded. We use the notation $\max _{d_{1}(x, y) \leq \epsilon}\left(d_{2}(x, y)\right)=K_{\epsilon}$. 
To find the elements $h_{1}$ and $h_{2}$ we choose an element $g \in G$ with large $\Delta(g)$. By Lemma 1.14 this means that the length of $\gamma_{g}$ is also large. Then we choose a point $y$ on the orbit of $x_{0}$ near the point on $\gamma_{g}$ where the function $\Delta$ becomes $\frac{1}{2} \Delta(g)$, which is still a large number. And then the geodesics connecting $y$ with $x_{0}$ and $g\left(x_{0}\right)$ pass near the geodesic $\gamma_{g}$ in different directions. Then they diverge fast comparative to $\Delta\left(y, x_{0}\right)$ and $\Delta\left(y, g\left(x_{0}\right)\right)$ which are close to $\frac{1}{2} \Delta(g)$.

Lemma 1.16. Under the conditions of the theorem, if for each $c \in \mathbb{R}$ there exists $g_{c} \in G$ such that $\Delta\left(g_{c}\right)>c$, then there are two elements $g_{1}$ and $g_{2}$ such that $\min \left(\Delta\left(g_{1}\right), \Delta\left(g_{2}\right)\right) \geq 4 f\left(g_{1}, g_{2}, 6 \delta\right)+20 \delta$.

This lemma finishes the proof of Theorem 1.1

\section{The Heisenberg group}

Let the discrete Heisenberg group $\Gamma$ act cocompactly on a space $M$ with respect to two metrics $d_{1}$ and $d_{2}$. Choose a point $x_{0} \in M$ and define metrics $d_{1}^{\prime}$ and $d_{2}^{\prime}$ on $\Gamma$ by the formulas

$$
\begin{aligned}
& d_{1}^{\prime}\left(\gamma_{1}, \gamma_{2}\right)=d_{1}\left(\gamma_{1}\left(x_{0}\right), \gamma_{2}\left(x_{0}\right)\right), \\
& d_{2}^{\prime}\left(\gamma_{1}, \gamma_{2}\right)=d_{2}\left(\gamma_{1}\left(x_{0}\right), \gamma_{2}\left(x_{0}\right)\right),
\end{aligned}
$$

for $\gamma_{1}, \gamma_{2} \in \Gamma$. The main result of this part of this work is the following theorem:

Theorem 2.1. Assume that

$$
\lim _{\max \left(d_{1}(x, y), d_{2}(x, y)\right) \rightarrow \infty} \frac{d_{1}(x, y)}{d_{2}(x, y)}=1
$$

and $d_{1}^{\prime}$ and $d_{2}^{\prime}$ are word metrics on $\Gamma$, then there is a constant $C$ such that $\mid d_{1}(x, y)-$ $d_{2}(x, y) \mid \leq C$ for each $x, y \in M$.

The proof of this theorem is based on the following statement from [7].

Statement 2.2. For each word metric on the Heisenberg group $\Gamma$, the GromovHausdorff distance between $\Gamma$ and its asymptotic cone $\mathrm{Con}_{\infty} \Gamma$ is finite.

2.1. Preliminaries. In this section, we give a description of the structure of the asymptotic cone of the Heisenberg group based on the results in [9].

The definition of the asymptotic cone and Gromov-Hausdorff distance can be found in [6]. Let us now describe the structure of the asymptotic cone of the Heisenberg group. To do this we need to introduce the notion of expansion.

Definition 2.3. The expansion of the group $\Gamma$ with coefficient $k$ is a map $\delta_{k}: \Gamma \rightarrow \Gamma$, $\delta_{k}(\gamma)=\left(k x, k y, k^{2} z\right)$, where $\gamma=(x, y, z), k \in \mathbb{Z}$.

The expansions $\delta_{k}$ of the group $\Gamma$ play the same role as homotheties in commutative groups. Let $\|\cdot\|: \Gamma \rightarrow \mathbb{R}$ be a norm on the group $\Gamma$, i.e., a real function that satisfies the properties:

- $\|\gamma\| \geq 0$ for each $\gamma \in \Gamma ;\|\gamma\|=0$ if and only if $\gamma=1$;

- $\|\gamma\|=\left\|\gamma^{-1}\right\|$

- $\left\|\gamma_{1} \gamma_{2}\right\| \leq\left\|\gamma_{1}\right\|\left\|\gamma_{2}\right\|$.

With each norm on $\Gamma$, one can associate a left-invariant metric on $\Gamma$

$$
\left|\gamma_{1}, \gamma_{2}\right|=\left\|\gamma_{1}^{-1} * \gamma_{2}\right\|
$$

We recall the definition of an interior metric on a discrete group given by Pansu [9]. 
Definition 2.4. The left-invariant metric associated with the norm $\|\cdot\|$ on the discrete group $G$ is called interior if for each $\varepsilon>0$ there exists $p>0$ such that $\forall \gamma \in G, \gamma$ can be represented as $\gamma=\gamma_{1} * \cdots * \gamma_{n}$. Here $\left\|\gamma_{i}\right\| \leq p$ and $\left\|\gamma_{1}\right\|+\cdots+$ $\left\|\gamma_{n}\right\| \leq(1+\varepsilon)\|\gamma\|$.

From Pansu's result [9], it follows that if the left-invariant metric associated with the given norm $\|\cdot\|$ on the Heisenberg group $\Gamma$ is interior, then the asymptotic cone of the Heisenberg group with this metric is the space $\left(H,\|\cdot\|_{\infty}\right)$, where $H$ is a continuous Heisenberg group. This is just the group of all upper triangular real $3 \times 3$ matrices with 1 's on the main diagonal. The left-invariant metric $d_{\infty}$ associated with the norm $\|\cdot\|_{\infty}$ is the Carnot-Carathéodory-Finsler metric given by the pair $\left(L_{0}, F_{0}\right)$; here $L_{0}$ is the two-dimensional subspace of the Lie algebra of the Lie group $H$, spanned by the vectors $(1,0,0)$ and $(0,1,0)$, and $F_{0}$ is the Minkowski metric on $L_{0}$. Moreover, for elements $\gamma \in \Gamma \subset H$ the $\operatorname{limit}_{\lim _{k \rightarrow \infty}} \frac{\left\|\delta_{k}(\gamma)\right\|}{k}$ exists and the following equality holds:

$$
\|\gamma\|_{\infty}=\lim _{k \rightarrow \infty} \frac{\left\|\delta_{k}(\gamma)\right\|}{k} .
$$

The number $\|\gamma\|_{\infty}$ is called the asymptotic norm of the element $\gamma \in \Gamma$.

Later, we will consider only the word metrics on the Heisenberg group $\Gamma$. Each such metric is associated with a finite generating set $E \subset \Gamma$, which we will call an alphabet. Each element $\gamma \in \Gamma$ can be represented as $\gamma=a_{1} * \cdots * a_{k}, a_{i} \in E$. The smallest such $k$ is called the length of the element $\gamma,\|\gamma\|=k$. It is obvious that the length is a norm. The corresponding left-invariant metric on $\Gamma$ is called the word metric. It is easy to see that the word metric is an interior metric on the Heisenberg group $\Gamma$.

Hereinafter, we identify $E$ with the subset of our lattice which in turn is embedded in $H$. Thus, we consider $E$ to be a subset of $H$. Let us also assume that $E$ is symmetric, i.e., $E=E^{-1}$. In particular, $|E|=2 n$ is an even number. A finite sequence of vectors of the alphabet is called a word. The number of vectors in a word is called the length of the word.

Let us reformulate Theorem 2.2. In order to prove the fact that

$$
\left|(\Gamma,\|\cdot\|) ;\left(H,\|\cdot\|_{\infty}\right)\right|_{H} \leq \text { Const }
$$

where $\|\cdot\|$ is a word norm on the Heisenberg group $\Gamma$, it is sufficient to compare two different norms $\|\cdot\|$ and $\|\cdot\|_{\infty}$ on $\Gamma$. If we prove that $\left|\|\gamma\|-\|\gamma\|_{\infty}\right| \leq$ Const for each $\gamma \in \Gamma$ and take an embedding $\Gamma \rightarrow H$, we will get the required statement. In other words, to prove Theorem [2.2, it is sufficient to check two inequalities:

$$
\begin{array}{r}
\|\gamma\|_{\infty} \leq\|\gamma\|+\text { Const } \\
\|\gamma\| \leq\|\gamma\|_{\infty}+\text { Const. }
\end{array}
$$

Let us now turn back to the metric spaces $\left(M, d_{1}\right)$ and $\left(M, d_{2}\right)$. Recall that the Heisenberg group $\Gamma$ acts cocompactly by isometries on both of them. We choose a point $x_{0} \in M$. This allows us to introduce two norms on the group $\Gamma$. Let $\gamma$ be an element of the group $\Gamma$. Then $\|\gamma\|_{1}=d_{1}\left(x_{0}, \gamma\left(x_{0}\right)\right)$ and $\|\gamma\|_{2}=d_{2}\left(x_{0}, \gamma\left(x_{0}\right)\right)$. These norms provide asymptotic norms $\|\cdot\|_{\infty 1}$ and $\|\cdot\|_{\infty 2}$ on the group $\Gamma$.

Lemma 2.5. Let $M$ be a set provided with two metrics $d_{1}$ and $d_{2}$ such that

$$
\lim _{\max \left(d_{1}(x, y), d_{2}(x, y)\right) \rightarrow \infty} \frac{d_{1}(x, y)}{d_{2}(x, y)}=1 .
$$


Let the Heisenberg group $\Gamma$ act by isometries with respect to both metrics. Then $\|\cdot\|_{\infty 1}=\|\cdot\|_{\infty 2}$.

Proof. Indeed, for each $\gamma \in \Gamma$ we have

$$
\|\gamma\|_{\infty 1}=\lim _{k \rightarrow \infty} \frac{\left\|\delta_{k}(\gamma)\right\|_{1}}{k}=\lim _{k \rightarrow \infty} \frac{\left\|\delta_{k}(\gamma)\right\|_{2}}{k} \frac{\left\|\delta_{k}(\gamma)\right\|_{1}}{\left\|\delta_{k}(\gamma)\right\|_{2}}=\lim _{k \rightarrow \infty} \frac{\left\|\delta_{k}(\gamma)\right\|_{2}}{k}=\|\gamma\|_{\infty 2}
$$

since $d_{2}\left(x_{0}, \delta_{k}(\gamma)\left(x_{0}\right)\right) \rightarrow \infty$ as $k \rightarrow \infty$.

Using (11) and (2) and the previous lemma we get that

$$
\begin{gathered}
\left|\|\gamma\|_{1}-\|\gamma\|_{\infty 1}\right| \leq \text { Const }_{1}, \\
\left|\|\gamma\|_{2}-\|\gamma\|_{\infty 2}\right| \leq \text { Const }_{2},
\end{gathered}
$$

and so

$$
\left|d_{1}\left(x_{0}, \gamma\left(x_{0}\right)\right)-d_{2}\left(x_{0}, \gamma\left(x_{0}\right)\right)\right| \leq \text { Const }_{1}+\text { Const }_{2} .
$$

Then it is enough to prove (1) and (2).

\section{Sketch of the proof of inequalities (1) And (2)}

In this section we describe the main idea of the proof of inequalities (11) and (2). The exact proof can be found in [7]. We start with a proof of (11) and (2) for word metrics of a special kind, which we now define.

Definition 3.1. If the third coordinates of all vectors of the alphabet $E$ are equal to 0 , then the alphabet is called horizontal and the corresponding word metric is called a horizontal word metric.

The inequality $\|\gamma\|_{\infty} \leq\|\gamma\|$ in the case of a horizontal word metric is an easy calculation. Indeed let the left-invariant metric on the Heisenberg group $\Gamma$ associated with the norm $\|\cdot\|$ be a horizontal word metric. Assume that $\gamma$ can be represented as $\gamma=a_{1} * \cdots * a_{N}$; then

$$
\delta_{k}(\gamma)=\delta_{k}\left(a_{1}\right) * \cdots * \delta_{k}\left(a_{N}\right)=a_{1}{ }^{k} * \cdots * a_{N}{ }^{k} .
$$

Therefore, $\left\|\delta_{k}(\gamma)\right\| \leq k N=k\|\gamma\|$ and

$$
\|\gamma\|_{\infty}=\lim _{k \rightarrow \infty} \frac{1}{k}\left\|\delta_{k}(\gamma)\right\| \leq N=\|\gamma\| .
$$

The proof of the other inequality is based on the following property of the Heisenberg group. Let $\gamma=(x, y, z)$ be an element of $\Gamma$. Assume that $\gamma=a_{1} * \ldots * a_{N}$, where $a_{i}$ are vectors of the horizontal alphabet $E$. Start at the origin and draw a closed polygonal line consisting of the vectors $a_{i}$ which lie in $O x y$ and the segment connecting the point $(x, y)$ with the origin. Then $|z|$ is equal to the oriented area enclosed in this polygonal line. This property follows from the formula for multiplication in the Heisenberg group: $\left(x_{1}, y_{1}, 0\right) *\left(x_{2}, y_{2}, 0\right)=\left(x_{1}+x_{2}, y_{1}+y_{2}, \frac{1}{2}\left(x_{1} y_{2}-y_{1} x_{2}\right)\right)$. The number $\frac{1}{2}\left(x_{1} y_{2}-y_{1} x_{2}\right)$ is equal to the area of the triangle with vertices $\left(x_{1}, y_{1}\right)$, $\left(x_{2}, y_{2}\right)$ and the origin.

This allows us to calculate the norm of an arbitrary element $\gamma=(x, y, z)$ in the horizontal word metric as the number of elements in the smallest collection of vectors of the alphabet on the plane $O x y$ which bounds the area $z$ and whose sum equals the vector $(x, y)$. This method of norm computation gives us an opportunity to reduce the proof of the inequalities (11) and (2) to the comparison of isoperimetric curves on the plane. 
Indeed, consider the Minkowski metric on the plane $O x y$, where the unit ball is the convex hull of the vectors of the horizontal alphabet $E$. The number of the vectors in some word is equal to the length of the corresponding polygonal line in this metric. Moreover, this metric, as follows from [1], is exactly the metric $F_{0}$ that gives rise to the Carnot-Carathéodory-Finsler metric on $\operatorname{Con}_{\infty} \Gamma$. So $\|\gamma\|_{\infty}$ is equal to the length of a certain path in the metric $F_{0}$. This path connects the points $(0,0)$ and $(x, y)$. It is also the shortest path that together with the segment from $(x, y)$ to $(0,0)$ bounds the area $z$. As follows from [8, Chapter 5, Theorem 22.4], this path is a polygonal line. The norm $\|\gamma\|$ is equal to the length of a certain polygonal line in the metric $F_{0}$. It connects the points $(0,0)$ and $(x, y)$. It is also the shortest polygonal line such that all segments in it are integer and it bounds the area $z$. These two polygonal lines are almost the same and the difference of their lengths is bounded by a constant. For details see [7].

To prove inequalities (11) and (2) for the case of general word metric we change the general alphabet $E=\left\{a_{1}=\left(x_{1}, y_{1}, z_{1}\right), \ldots, a_{2 n}=\left(x_{2 n}, y_{2 n}, z_{2 n}\right)\right\}$ to the horizontal alphabet $E_{0}=\left\{\left(x_{1}, y_{1}, 0\right), \ldots,\left(x_{2 n}, y_{2 n}, 0\right)\right\}$. Then we mention that the corresponding word metrics differ by a constant. To see this we check that the polygonal line (of projections of the alphabet vectors on $O x y$ ) corresponding to some almost the shortest word, which gives us some element $\gamma=(x, y, z)$, consists of at most $2 n+2$ segments. That means that the same vectors come together in the shortest word. Then we consider each segment of the polygonal line. It consists of $r_{i}$ projections on $O x y$ of vectors $a_{i}$. Change it for three segments that finish a parallelogram whose area is proportional to $z_{i}$ and $r_{i}$. We will choose this parallelogram in such a way that the area bounded by the new curve is $z$. Then the length of the new polygonal line is almost the norm of $\gamma$ in a horizontal word metric with alphabet $E_{0}$. And it differs from the length of the initial polygonal line by a constant, depending only on the alphabet.

\section{REFERENCES}

[1] Berestovskii, V. N., Geodesics of nonholonomic left invariant interior metrics on the Heisenberg group and isoperimetrics on the Minkowski plane, Siberian Math. J. 35 (1994), no. 1, 1-8. MR 95m:58034

[2] Burago, D., Periodic metrics, Representation Theory and Dynamical Systems, pp. 205-210, Adv. Soviet Math., vol. 9, Amer. Math. Soc., Providence, RI, 1992. MR 93c:53029

[3] Buyalo, S. V., Introduction to the metric geometry, St. Petersburg, Obrazovanie, 1997.

[4] Gromov, M., Carnot-Carathéodory spaces seen from within, Sub-Riemannian Geometry, pp. 79-323, Progr. Math., vol. 144, Birkhäuser, Basel, 1996. MR 2000f:53034

[5] Gromov, M., Asymptotic invariants of infinite groups, Geometric Group Theory, Vol. 2 (G. A. Noble, M. A. Roller, eds.), London Math. Soc. Lecture Notes Ser., vol. 182, Cambridge Univ. Press, Cambridge, 1993. MR 95m:20041]

[6] Gromov, M., Structures métriques pour les variétés riemanniennes (J. Lafontaine et P. Pansu, eds.), Cedic/Fernand Nathan, Paris, 1981. MR 85e:53051

[7] Krat, S. A., Asymptotic properties of the Heisenberg group, Zap. Nauchn. Seminar. POMI, vol. 261, 1999, pp. 125-154. CMP 2000:12

[8] Leichtweiss, K., Konvexe Mengen, Hochschultext, Springer-Verlag, Berlin-New York, 1980. MR 81b:52001

[9] Pansu, P., Croissance des boules et des géodésiques fermées dans les nilvariétés, Ergodic Theory Dynam. Systems 3 (1983), 415-445. MR 85m:53040

Department of Mathematics, The Pennsylvania State University, University Park, PA 16802

E-mail address: krat@math.psu.edu 